\title{
Unusually Wide, High-Velocity Radio Recombination Lines from G0.15-0.05 in the Radio Arc
}

\author{
F. Yusef-Zadeh ${ }^{1}$ \\ LASP, Goddard Space Flight Center and Northwestern University \\ Mark Morris \\ Astronomy Department, University of California, Los Angeles \\ and J. H. van Gorkom \\ Columbia University and National Radio Astronomy Observatory
}

\begin{abstract}
The $\mathrm{H} 92 \alpha$ recombination line was observed at $8 \mathrm{GHz}$ toward the "pistolshaped" HII region G0.15-0.05 using the VLA ${ }^{2}$ in its most compact configuration. The line profiles of individual components of this source peak at $\mathrm{V}_{L S R}=123 \mathrm{~km} / \mathrm{s}$ and have total line widths of $\sim 90 \mathrm{~km} / \mathrm{s}$. The kinematical structure of the "pistol" is unusual in that much of the neutral and ionized gas in this region is seen predominantly at either +50 or $+20 \mathrm{~km} / \mathrm{s}$. The line width and radial velocity are the largest found in the Galactic center region with the exception of Sgr A West. We also found gas at $\mathrm{V}_{L S R}=140 \mathrm{~km} / \mathrm{s}$ associated with G0.18-0.04: the sickle-shaped feature which surrounds G0.15-0.05. The kinematic properties of G0.18-0.04 and G0.15-0.05 suggest that these two features are components of a single, but complex thermal system interacting with the nonthermal filaments of the radio Arc. In this regard, the width of the broad recombination line from G0.15-0.05, and its large radial velocity, might be explained as the interaction of streaming relativistic particles in the nonthermal filaments of the Arc impacting upon ambient gas clouds lying in the Galactic plane.
\end{abstract}

\section{Introduction}

The radio continuum Arc located near the Galactic center at $1 \sim 0.2^{\circ}$ is known to consist of both thermal and nonthermal structures (Yusef-Zadeh et al. 1986). One of the prominent thermal features associated with the radio Arc lies at the region where the linear filaments cross the Galactic plane. Here, we present recombination line maps of this region and discuss the kinematics of ionized gas associated with the compact source G0.15-0.05. High-resolution radio continuum observations show that the "pistol-shaped" structure G0.15-0.05 has considerable substructure, including at least 6 compact peaks (Yusef-Zadeh and Morris 1987a). Mid-infrared observations indicate that this object has a large ratio of IR to radio flux densities and that it lies adjacent to a group of compact IR sources having an unusually high dust color temperature (see Okuda et al.; Yusef-Zadeh, Telesco and Decher, this conference).

1 NRC National Research Associate

2 VLA is a component of the National Radio Astronomy Observatory under contract to the National Science Foundation 

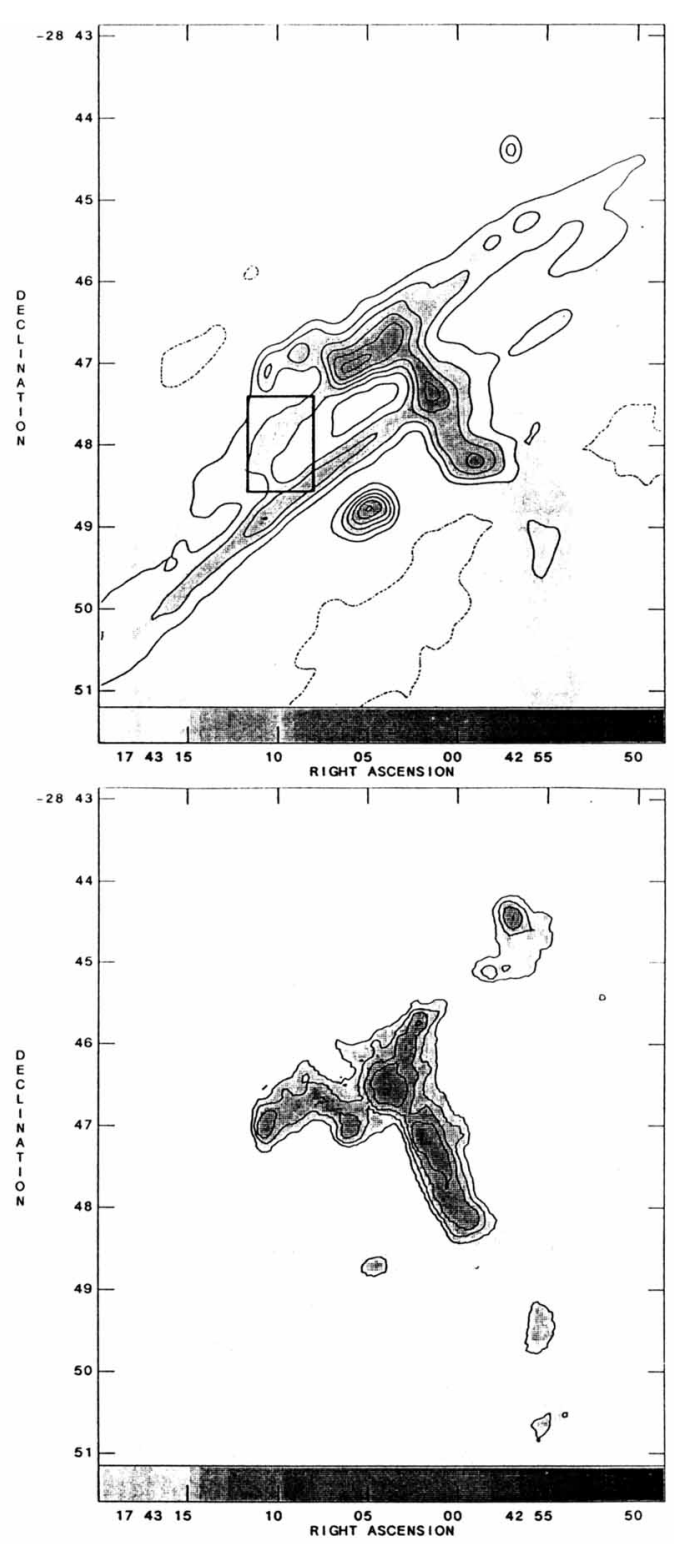

Figure 1a,b: [top] Continuum emission at $\lambda 6 \mathrm{~cm}$ from the region where the Arc crosses the Galactic plane. This region consists of two thermal components, G0.18-0.04 and G0.15-0.05. Contour levels are set at $-30,30,60,90,120,150$ and $180 \mathrm{mJy} /$ beam area. No correction has been applied to account for the response of the primary beam. Fig. 4 gives the spectrum arising within the rectangular box drawn on this figure. [bottom] The H110 $\alpha$ integrated line emission from the same region, with contour levels set at $50,100,150$, and $200 \mathrm{~km} / \mathrm{s} \times \mathrm{mJy} /$ beam area. 


\section{Observations and Results}

Recombination line emission at $\lambda 6 \mathrm{~cm}(\mathrm{H} 110 \alpha)$ from the vicinity of G0.180.04 was observed with the hybrid C/D configuration of the VLA (Yusef-Zadeh 1986). The spatial and velocity resolutions were $16.1 " \times 12.6 "(\delta \times \alpha)$ and $6 \mathrm{~km} / \mathrm{s}$, respectively. Figure 1a, which is constructed from the continuum channel at $5 \mathrm{GHz}$, shows the continuum emission at this resolution from the region surrounding G0.180.04 . The linear filaments, which are oriented in the direction perpendicular to the Galactic plane, are crossed by a sickle-shaped structure, G0.18-0.04. In addition, a bright compact object, G0.15-0.05, can be seen at $\alpha=17^{h} 42^{m} 4.7^{s}, \delta=-28^{\circ} 48^{\prime}$ 42 ". Figure 1b shows the total $\mathrm{H} 110 \alpha$ line intensity $(\mathrm{H} 110 \alpha)$ and indicates that all the line emission arises from G0.18-0.04 and G0.15-0.05. The lack of thermal emission from the linear filaments is consistent with nonthermal characteristics inferred from polarization measurements (Yusef-Zadeh and Morris 1987b; Inoue et $a l .$, this conference). Much of the ionized gas along the sickle has positive radial velocity, centered predominantly between 30 and $40 \mathrm{~km} / \mathrm{s}$, and typical line widths of $\sim 20 \mathrm{~km} / \mathrm{s}$ (Yusef-Zadeh 1986). In this $\mathrm{H} 110 \alpha$ measurement, we noted that the line emission from G0.15-0.05 peaks at or beyond edge of the observed velocity range, $110 \mathrm{~km} / \mathrm{s}$.

In July 1988 , we reobserved G0.18-0.04 at $8.3 \mathrm{GHz}(\mathrm{H} 92 \alpha)$ using the VLA in its " $\mathrm{D}$ " configuration in order to obtain the complete kinematic information on G0.150.05 . We used 64 channels with a center velocity of $+60 \mathrm{~km} / \mathrm{s}$; the total bandwidth was $12.5 \mathrm{MHz}$, giving a velocity resolution of $7 \mathrm{~km} / \mathrm{s}$. The spatial resolution of the $8 \mathrm{GHz}$ maps, 20 " $\times 10.3$ ", is similar to that of $\lambda 6 \mathrm{~cm}$ maps shown in Figure 1 . Because of the low system temperature of $8 \mathrm{GHz}$ receivers $\left(30^{\circ} \mathrm{K}\right)$, the rms noise in the channel maps presented here $(0.6 \mathrm{mJy})$ is a factor of 2 better than that of the $\lambda 6 \mathrm{~cm}$ measurements, as previously described by Yusef-Zadeh (1986). The sensitivity of these measurements has been particularly essential in clarifying the overall kinamatical structure, and the relationship of G0.18-0.04 and G0.15-0.05. Here, we present the preliminary results of the $8 \mathrm{GHz}$ observation and postpone a more detailed account of both $\mathrm{H} 110 \alpha$ and $\mathrm{H} 92 \alpha$ observations to future publications.

The integrated H92 $\alpha$ line profile from a 30" region centered G0.15-0.05 is shown in Figure 2; it shows a center velocity of $\mathrm{V}_{L S R} \sim 120 \mathrm{~km} / \mathrm{s}$ and a total line width of $\sim 90 \mathrm{~km} / \mathrm{s}$. A selected number of channel maps (Figure 3) show the complex velocity field associated with this source and indicate a velocity gradient of $\sim 2$ $\mathrm{km} / \mathrm{s} /$ arcsecond across it. Although high-resolution radio continuum maps show that the "pistol" consists of a number of compact sources, the channel maps clearly indicate that much of the broad line emission arises from the region where free-free emission is generally weak and diffuse. In fact, the ionized gas with the largest center velocities does not coincide with any of the discrete peaks tabulated by Yusef-Zadeh and Morris (1987a). We cannot, however, rule out the possibility that several spatially unresolved components with smaller line widths contribute to the formation of the observed large line widths.

Using the observed line to continuum ratio, we estimate that $\mathrm{G} 0.15-0.05$ has an LTE electron temperature which is roughly $\leq 6.2 \times 10^{3} \mathrm{~K}$, assuming that the ionized plasma is homogeneous and is in Local Thermodynamic Equilibrium (see Garay, Rodriguez and van Gorkom 1986). Comparison of $\lambda 6 \mathrm{~cm}$ and $\lambda 3.6 \mathrm{~cm}$ continuum 


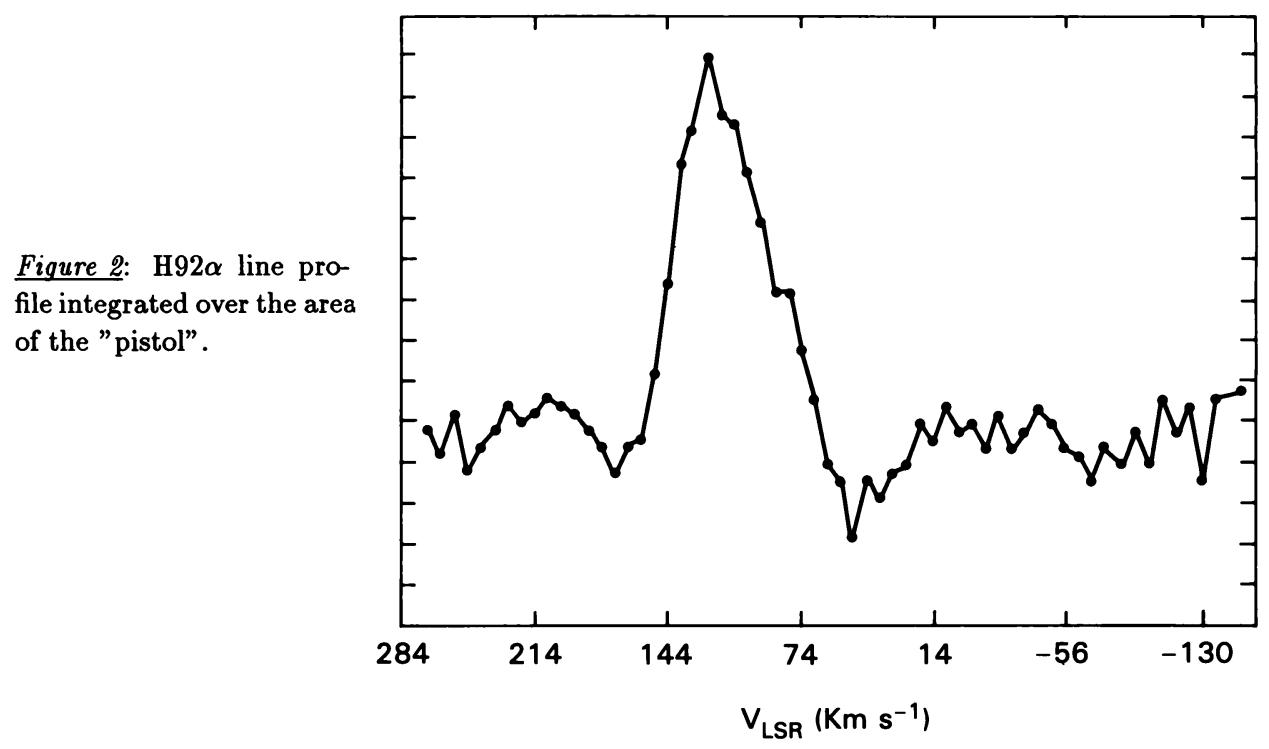

Figure 3(a-d): Selected channel maps showing H92 $\alpha$ emission from the "pistol". Contour levels are set at $-1,1$, $1.5,2,2.5,3,4,5,6,7$, $8,9,10,12,14 \mathrm{mJy} /$ beam area. The central velocity of each channel is indicated in the box.

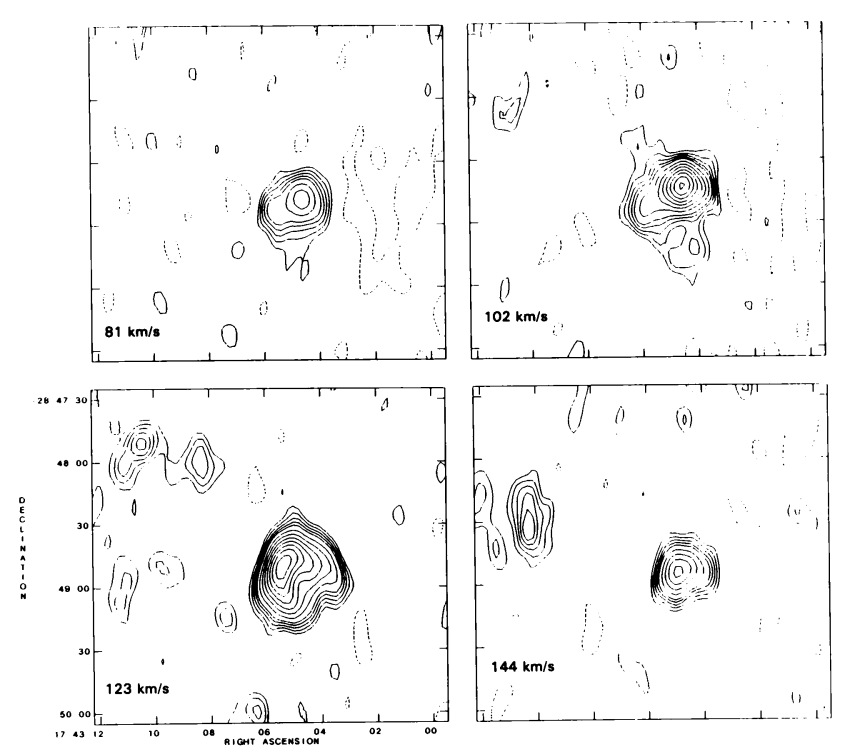


emission indicates that the "pistol" has a spectrum which is very consistent with free-free emission from optically thin ionized plasma. The basic parameters of the HII region can be estimated by assuming that the source is a homogeneous sphere of diameter $30^{\prime \prime}$, an electron temperature of $10^{4}{ }^{\circ} \mathrm{K}$, and a total flux of $\sim 0.6 \mathrm{Jy}$ at 4.87 GHz (Schraml and Mezger 1969; Rodriguez et al. 1980). Thus at an assumed distance of $8.5 \mathrm{kpc}$, the electron density is $\sim 400 \mathrm{~cm}^{-3}$ and the total amount of ionized gas is estimated to be $\sim 32 \mathrm{M}_{\odot}$. The number of ionizing photons needed to ionize the gas is $\sim 3.9 \times 10^{48} \mathrm{~s}^{-1}$.

\section{Discussion}

The similarity noted in the velocity structure of both G0.15-0.05 and G0.180.04 suggests that they are physically associated with each other and that the ionization of these two features may be produced as a result of collision between relativistic particles in the nonthermal filaments of the radio Arc and ambient gas clouds along the Galactic plane (Yusef-Zadeh and Morris 1987a). The basis for the latter argument lies in the exceptionally high center velocity and velocity dispersion of ionized gas in this region, whereas the bulk of the molecular and atomic gas are associated with either the $40 \mathrm{~km} / \mathrm{s}$ cloud or the $20 \mathrm{~km} / \mathrm{s}$ cloud (Brown and Liszt 1984; Bally et al. 1988; Lasenby et al., this conference). Future high-resolution molecular line observations should identify the particular molecular features with which G0.15-0.05 and G0.18-0.04 are associated. In addition, the linear filaments are distorted to the east of the "pistol" and they undergo an apparent discontinuity at the location where the "pistol" is situated (see Figure 2 of Yusef-Zadel and Morris 1987b); these structural details were used to argue for the interaction hypothesis. Finally, the large intensity ratio of IR to radio in the pistol and the presence of a cluster of unusual IR sources, known as the quintuplet, imply indirectly that unusual heating of the dust grains may be taking place in this region (Yusef-Zadeh, Telesco and Decher 1988).

Because of the relatively small density of ionized gas in the "pistol", pressure broadening can not be the cause of the line breadth. The large line width may be best explained in terms of a microturbulent velocity as large as 25 to $30 \mathrm{~km} / \mathrm{s}$. This mechanism has also been proposed previously to explain the anomalously large line widths in ultracompact HII regions having a cometary morphology and the large line width seen in Sgr A West (Garay, Rodriguez and van Gorkom 1986; van Gorkom et al. 1988). We suggest that the origin of the turbulence in G0.15-0.05 is the bulk streaming motion of relativistic particles impacting dense neutral clouds.

MM acknowledges support from NSF grant AST87-18068 to UCLA.

\section{References}

Bally, J. Stark, T., Wilson, R.W. and Henkel, C. 1988 Ap.J., 324, 223. Brown, R.L. and Liszt, H.S. 1984 Ann.Rev.Ast.Ap., 22, 223.

Garay, G., Rodriguez, L.F. and van Gorkom, J.H. 1986 Ap.J., 309, 553.

Okuda, H., Shibai, H., Nakagawa, T., Matsuhara, H., Kobayashi, Y. et al. 1988, Presented at the IAU Symposium no. 196 on the Galactic Center. 
Rodriguez, L.F., Moran, J.M., Ho, P.T.P. and Gottlieb, E.W. 1980, Ap.J., 235, 845 .

Schraml, J. and Mezger, P. G. 1969, Ap.J., 156, 269.

van Gorkom, J.H., Schwarz, U.J. and Bregman, J.D. 1988

Presented at the IAU Symposium no. 136 on the Galactic Center.

Yusef-Zadeh, F. 1986, Ph.D Thesis, Columbia University.

Yusef-Zadeh, F. and Morris, M. 1987a, Astron.J. 94, 557.

Yusef-Zadeh, F. and Morris, M. 1987b, Ap. J. 322, 721.

Yusef-Zadeh, F., Morris, M., Slee, O.B. and Nelson, G. J. 1986, Ap.J., 310, 689 .

Yusef-Zadeh, F., Telesco, C.M. and Decher, B. 1988, Presented at the IAU Symposium no. 136 on the Galactic Center.

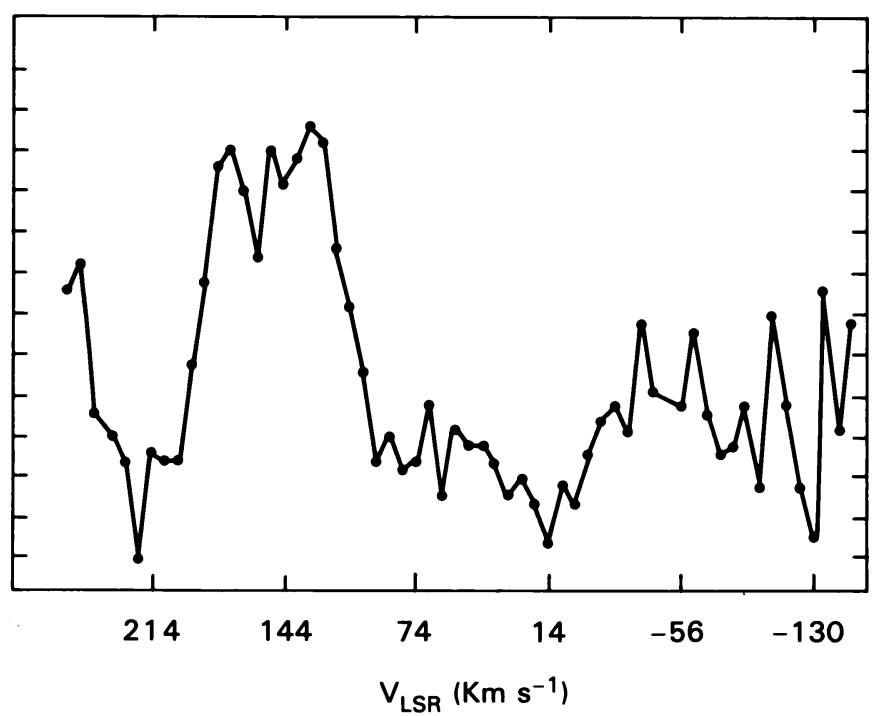

Figure $4: \mathrm{H} 29 \alpha$ line profile integrated over the rectangular box drawn in Figure $1 \mathrm{a}$. 